ANL/ET/PP- 82651 ULTRASONIC WAVE PROPAGATION IN MULTILAYERED PIEZOELECTRIC SUBSTRATES*

H.-T. Chien, S.-H. Sheen, and A. C. Raptis PECENED

121396

OSTI

\author{
Energy Technology Division \\ ARGONNE NATIONAL LABORATORY \\ Argonne, Illinois 60439
}

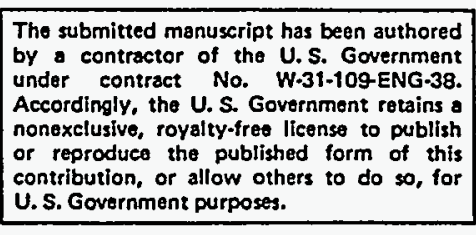

.

April 11, 1994

To be presented in the Special Issue on Thin-films for Acoustoelectronics of IEEE Transactions on Ultrasonics, Ferroelectrics, and Frequency Control.

"Work sponsored by the U.S. Department of Energy, Arms Control, and Nonproliferation, Advanced Concept Programs.

DLSTRIESTION OF FHIS DOCLRENT IS UNG

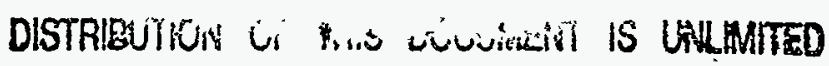




\section{DISCLAIMER}

Portions of this document may be illegible in electronic image products. Images are produced from the best available original document. 


\title{
Ultrasonic Wave Propagation in Multilayered Piezoelectric Substrates ${ }^{1}$
}

\author{
H.-T. Chien, S.-H. Sheen, and A. C. Raptis ${ }^{2}$
}

Abstract-Due to the increasing demand for higher operating frequency, lower attenuation, and stronger piezoelectricity, use of the layered structure has become necessary. Theoretical studies are carried out for ultrasonic waves propagating in the multilayered piezoelectric substrates. Each layer processes up to as low as monoclinic symmetry with various thickness and orientation. A plane acoustic wave is assumed to be incident, at varied frequency and incidence angle, from a fluid upon a multilayered substrate. Simple analytical expressions for the reflection and transmission coefficients are derived from which all propagation characteristics are identified. Such expressions contain, as a by-product, the secular equation for the propagation of free harmonic waves on the multilayered piezoelectric substrates. Solutions are obtained for the individual layers which relate the field variables at the upper layer surfaces. The response of the total system proceeds by satisfying appropriate interfacial conditions across the layers. Based on the boundary conditions, two cases, "shorted" and "free", are derived from which a so-called piezoelectric coupling factor is calculated to show the piezoelectric efficiency. Our results are rather general and show that the phase velocity is a function of frequency, layer thickness, and orientation.

\footnotetext{
${ }^{1}$ Work sponsored by the U.S. Department of Energy, Arms Control and Nonproliferation, Advanced Concept Programs.

${ }^{2}$ The authors are with the Energy Technology Division, Argonne National Laboratory, Argonne, IL 60439 .
} 


\section{INTRODUCTION}

In previous papers [1], [2], Nayfeh and Chien presented a unified analytical treatment for the interactions of ultrasonic waves in piezoelectric plates and substrates. The media are allowed to posses as low as monoclinic symmetry and associated piezoelectric coupling. They are also assumed to be immersed in water and subjected to inclident acoustic beams at arbitrary polar and azimuthal angles. Simple analytical expressions for the reflection and transmission coefficients were derives from which all propagation characteristics are identified. Such expressions contain, as a by-product, the secular equation for the propagation of free harmonic waves on the piezoelectric media. Higher symmetries, such as orthotropic, transverse isotropic and cubic, are contained implicitly in this analysis.

Because that the wave vectors of the incident and reflected waves all lie on the same plane [3], a linear transformation can be applied to simplify the analysis and to facilitate compact expressions of the final results. Previously, Nayfeh has successfully applied the general approach utilized in [4] to develop solutions for the interactions of ultrasonic waves with a wide variety of single and multilayered plate media in the absence of piezoelectric effects. Techniques utilized in [4] and [5] have also been employed to develop solutions for a variety of multilayered anisotropic media [6]. A unified analytical treatment supported by extensive experimental data of the interaction of ultrasonic waves with an arbitrarily oriented orthotropic elastic plate [7], [S].

The purpose of this paper is to extend the analysis of a single piezoelectric media to the case of a multilayered substrate, which consists an arbitrary number of piezoelectric layers rigidly bonded at their interfaces on a solid half-space. The analysis will be general and can be handle layers of either different materials or layers of the same materials with different orientations. Solutions will be obtained by utilizing the single plate's formal solutions together with the matrix transfer method [1]. Using the matrix transfer method, the reflection and transmission coefficients of the total system will are derived and from which all of the propagation characteristics 
are readily extracted. The generic difficulties of the mathematical analysis of waves in anisotropic media as compared with those pertaining to isotropic media are fully discussed (included extensive literature review) in [1], [2], and [4]-[8], and need not to be elaborated upon here.

\section{GOVERNING FIELD EQUATION OF PIEZO- ELECTRICITY}

The linear theory of piezoelectricity introduces linear coupling between the acoustic field equations and Maxwell's electromagnetic field equations and governs the characteristics of wave propagation in a piezoelectric medium [9], [10]. The linear piezoelectric constitutive equations, derived from the linear theory of piezoelectricity, of a piezoelectric medium are

$$
\begin{aligned}
& T_{i j}=C_{i j k \ell} S_{k \ell}-e_{k i j} E_{k} \\
& D_{k}=e_{k i j} S_{i j}+\epsilon_{k i} E_{i}
\end{aligned}
$$

where $T_{i j}$ represents the stress, $C_{i j k \ell}$ the elastic stiffness constant, $S_{i j}=1 / 2\left(\partial u_{i} / \partial x_{j}+\right.$ $\left.\partial u_{j} / \partial x_{i}\right)$ the strain, $u_{i}$ the mechanical displacement, $e_{k i j}$ the piezoelectric stress constant, $E_{k}=-\partial \phi / \partial x_{k}$ the electric field, $\phi$ a scalar electric potential, $D_{i}$ the electric displacement, $\epsilon_{k i}$ the dielectric permittivity, $x_{i}=\left(x_{1}, x_{2}, x_{3}\right)$ the coordinate system, and $i, j, k, \ell=1,2,3$. The coupled piezoelectric field equations are given by the motion equations and the electrostatic charge. The linear electrostatic piezoelectric equations are derived as

$$
\begin{aligned}
C_{i j k \ell} u_{k, j \ell}+e_{k i j} \phi_{, k j} & =\rho \ddot{u}_{i} \\
e_{k i j} u_{i, j k}-\epsilon_{k i} \phi_{, i k} & =0
\end{aligned}
$$

where $\rho$ is the material density. The elastic stiffness constants $\left(C_{i j k l}\right)$, the piezoelectric stress constants $\left(e_{i j k}\right)$, and the dielectric permittivities $\left(\epsilon_{i j}\right)$ are fourth, third and second rank tensors, respectively, and follow the tensor transformation [10]. The summation convention applies to equations (1) and (2). 
The field equation and the constitutive equation of a fluid are

$$
T_{i j, j}^{f}=\rho_{f} \ddot{u}_{i}^{f}
$$

and

$$
T_{i j}^{f}=\lambda_{f} u_{k, k}^{f} \delta_{i j}
$$

respectively, where $\rho_{f}$ represents the fluid density, $\lambda_{f}$ the fluid bulk modulus, and $\delta_{i j}$ the Kronecker delta.

\section{FORMULATION OF THE PROBLEM}

Consider a multilayered substrate consisting of an arbitrary number, $n$, of piezoelectric layers rigidly bonded at their interfaces on a solid half-space. Layers are stacked normal to the $x_{3}$-axis of a global orthogonal Cartesian system $x_{i}=\left(x_{1}, x_{2}, x_{3}\right)$. Hence the plane of each layer is parallel to the $x_{1}-x_{2}$ plane which is also chosen to coincide with the bottom surface of the layered substrate. In order to maintain generality we shall assume each layer to be arbitrarily oriented in the $x_{1}-x_{2}$ plane. To describe the relative orientation of the layers we shall assign for each layer $k, k=1,2, \ldots, n$, a local Cartesian coordinate $\left(x_{1}^{\prime}\right)_{k}$ coinciding with its axis of symmetry such that its origin is located in the middle plane of the layer with $\left(x_{3}^{\prime}\right)_{k}$ normal to it. Thus layer $k$ extends from $-d_{k} / 2 \leq\left(x_{3}^{\prime}\right)_{k} \leq d_{k} / 2$, where $d_{k}$ is its thickness. The total thickness of the layers is $d$. Equivalently, the orientation of the $k$ th layer in the $x_{i}$ space can be described by a rotation of an angle $\psi_{k}$ between $\left(x_{1}^{\prime}\right)_{k}$ and $x_{1}$. Once all orientation angles $\psi_{k}$ are specified the geometry of the layered substrate will be defined. The geometry and modeling of the multilayered substrate is shown in Fig. 1.

Without any loss in generality we shall assume that a plane wave is incident in the $x_{1}-x_{2}$ plane on the medium from the upper fluid at an arbitrary angle $\theta$. The problem here is to study the reflected and transmitted fields. A key condition which is found to facilitate our subsequent analysis is the fact that the wave vectors of the incident and refracted waves must all lie in the same plane [3]. This is a consequence of the continuity conditions at the interfaces. We therefore conduct our 
analysis in a coordinate system formed by incident and reflected planes rather than by material symmetry axes. Accordingly, the primed system $\left(x_{i}^{\prime}\right)_{k}$ rotates with one material symmetry axis while the global unprimed system $x_{i}$ remains invariant. This approach leads to significant simplification in our algebraic analysis and computations [1].

In this section we follow the analytical procedure of [1] to construct a transfer matrix for each layer $k$. In order to derive the reflection and transmission coefficients, the field equations of fluid must also be given in a manner similar to those of solid. In our problem, we shall assume that the fluid (water) does not support piezoelectric effects and hence its electric potential is zero. Accordingly, there will be no change in the fluid's field equations or their formal solutions from those given in our earlier work [1], [2]. For this reason we shall only quote such material from them later on in this analysis.

Formally, we can proceed to analyze the most general anisotropic medium (the triclinic one) for each layer which has 21 elastic constants, 9 dielectric permittivities, and 18 coupling coefficients. The expressions will be algebraically complicated and their utility will be numerically limited as was pointed out in [1]. For the slightly more symmetric materials, i.e., the monoclinic ones, dramatic simplifications can be achieved for the final expressions. Therefore, we shall limit the following analysis to monoclinic materials.

Anisotropic medium with one plane of material symmetry is termed monoclinic. Two classes of such materials exist: these belong to an " $m$ " or " 2 " groups whose constitutive relations are respectively shown in expanded matrix manner as the following 


$$
\left\{\begin{array}{l}
T_{11} \\
T_{22} \\
T_{33} \\
T_{23} \\
T_{13} \\
T_{12} \\
D_{1} \\
D_{2} \\
D_{3}
\end{array}\right\}=\left[\begin{array}{ccccccccc}
C_{11} & C_{12} & C_{13} & 0 & 0 & C_{16} & 0 & 0 & e_{31} \\
C_{12} & C_{22} & C_{23} & 0 & 0 & C_{26} & 0 & 0 & e_{32} \\
C_{13} & C_{23} & C_{33} & 0 & 0 & C_{36} & 0 & 0 & e_{33} \\
0 & 0 & 0 & C_{44} & C_{45} & 0 & e_{14} & e_{24} & 0 \\
0 & 0 & 0 & C_{45} & C_{55} & 0 & e_{15} & e_{25} & 0 \\
C_{16} & C_{26} & C_{36} & 0 & 0 & C_{66} & 0 & 0 & e_{36} \\
0 & 0 & 0 & e_{14} & e_{15} & 0 & \epsilon_{11} & \epsilon_{12} & 0 \\
0 & 0 & 0 & e_{24} & e_{25} & 0 & \epsilon_{12} & \epsilon_{22} & 0 \\
e_{31} & e_{32} & e_{33} & 0 & 0 & e_{36} & 0 & 0 & \epsilon_{33}
\end{array}\right] \quad\left\{\begin{array}{c}
S_{11} \\
S_{22} \\
S_{33} \\
S_{23} \\
S_{13} \\
S_{12} \\
E_{1} \\
E_{2} \\
E_{3}
\end{array}\right\}
$$

and

$$
\left.\left\{\begin{array}{l}
T_{11} \\
T_{22} \\
T_{33} \\
T_{23} \\
T_{13} \\
T_{12} \\
D_{1} \\
D_{2} \\
D_{3}
\end{array}\right\}=\left[\begin{array}{ccccccccc}
C_{11} & C_{12} & C_{13} & 0 & 0 & C_{16} & e_{11} & e_{21} & 0 \\
C_{12} & C_{22} & C_{23} & 0 & 0 & C_{26} & e_{12} & e_{22} & 0 \\
C_{13} & C_{23} & C_{33} & 0 & 0 & C_{36} & e_{13} & e_{23} & 0 \\
0 & 0 & 0 & C_{44} & C_{45} & 0 & 0 & 0 & e_{34} \\
0 & 0 & 0 & C_{45} & C_{55} & 0 & 0 & 0 & e_{35} \\
C_{16} & C_{26} & C_{36} & 0 & 0 & C_{66} & e_{16} & e_{26} & 0 \\
e_{11} & e_{12} & e_{13} & 0 & 0 & e_{16} & \epsilon_{11} & \epsilon_{12} & 0 \\
e_{21} & e_{22} & e_{23} & 0 & 0 & e_{26} & \epsilon_{12} & \epsilon_{22} & 0 \\
0 & 0 & 0 & e_{34} & e_{35} & 0 & 0 & 0 & \epsilon_{33}
\end{array}\right] \quad \begin{array}{c}
S_{11} \\
S_{22} \\
S_{33} \\
S_{23} \\
S_{13} \\
S_{12} \\
E_{1} \\
E_{2} \\
E_{3}
\end{array}\right\}
$$

In these expanded forms we used the contracting subscript notation $1 \rightarrow 11$, $2 \rightarrow 22,3 \rightarrow 33,4 \rightarrow 23,5 \rightarrow 13$, and $6 \rightarrow 12$ to relate $C_{p q}$ and $e_{k p}$ to $C_{i j k \ell}$ and $e_{k i j}$, respectively $(p, q=1,2, \ldots, 6$ and $i, j, k, \ell=1,2,3)$. Thus, $C_{25}$ stands for $C_{2213}$ and $e_{14}$ stands for $e_{123}$, for examples. Notice that the purely elastic or electric portions of these relations are identical whereas the coupled portions are different. In fact, by further examination we conclude that the vanishing entries in one correspond to the nonvanishing entries of the other; i.e., there are no common nonvanishing coupling terms. As will be shown later, such unique properties have important consequences in the manner in which the various waves interact. For this reason we need to treat both cases separately. It is expected that, upon presenting solutions for one case, results for the second case will be identified by inspection. Accordingly, we shall proceed to first analyze the case of monoclinic-2 case.

\subsection{MONOCLINIC-2 CLASS}

The particle motion generally have three nonzero spatial components $u_{1}, u_{2}$, and $u_{3}$ corresponding to longitudinal wave $(P)$ along the $x_{1}$-axis, horizontally and vertically 
polarized transverse waves ( $S H$ and $S V$ ), respectively, and the electric potential $\phi$. In the absence of material symmetry these three waves will couple together and be stiffened lending to a complicated response. For plane waves propagating along the $x_{1}$-axis and independent of $x_{2}$-axis, a formal solution for $u_{i}$ and $\phi$ can be written as

$$
\left\{u_{1}, u_{2}, u_{3}, \phi\right\}=\bar{U} \exp ^{i \xi\left(x_{1}+\alpha x_{3}-c t\right)}
$$

where $i=\sqrt{-1}, \xi$ is the wave number, $\omega$ is the circular frequency, $c$ is the phase velocity $(=\omega / \xi), \alpha$ is still an unknown parameter. $\bar{U}=\{U, V, W, \Phi\}$ are constant displacement amplitudes. Combinations of equations (7) and (2) yield four linear homogeneous coupled equations

$$
\Gamma_{m n}(\alpha) U_{n}=0, \quad m, n=1,2,3,4
$$

where the summation convention holds and $\Gamma_{n m}=\Gamma_{m n}$ :

$$
\begin{array}{ll}
\Gamma_{11}(\alpha)=C_{11}-\rho c^{2}+C_{55} \alpha^{2} & \Gamma_{12}(\alpha)=C_{16}+C_{45} \alpha^{2} \\
\Gamma_{22}(\alpha)=C_{66}-\rho c^{2}+C_{44} \alpha^{2} & \Gamma_{13}(\alpha)=\left(C_{13}+C_{55}\right) \alpha \\
\Gamma_{33}(\alpha)=C_{55}-\rho c^{2}+C_{33} \alpha^{2} & \Gamma_{23}(\alpha)=\left(C_{36}+C_{45}\right) \alpha \\
\Gamma_{14}(\alpha)=\left(e_{15}+e_{31}\right) \alpha & \Gamma_{34}(\alpha)=e_{15}+e_{33} \alpha^{2} \\
\Gamma_{24}(\alpha)=\left(e_{14}+e_{36}\right) \alpha & \Gamma_{44}(\alpha)=-\epsilon_{11}-\epsilon_{33} \alpha^{2} .
\end{array}
$$

Nontrivial solutions for $U_{n}$ demand the vanishing of the determinant in equation (8) and yield an eighth-degree polynomial equation in $\alpha$ as

$$
\alpha^{8}+A_{1} \alpha^{6}+A_{2} \alpha^{4}+A_{3} \alpha^{2}+A_{4}=0
$$

which is a characteristic equation relating $\alpha$ to $c$ and whose coefficients $A_{1}, A_{2}, A_{3}$, and $A_{4}$ are listed in Appendix A of [2]. Equation (10) now admits four solutions for $\alpha^{2}$ and leads to eight solutions for $\alpha$ which are restricted such that

$$
\alpha_{2}=-\alpha_{1}, \quad \alpha_{4}=-\alpha_{3}, \quad \alpha_{6}=-\alpha_{5}, \quad \alpha_{8}=-\alpha_{\bar{T}} .
$$

For each $\alpha_{q}$ we can use equation (S) to relate the amplitude ratios as

$$
\begin{aligned}
V_{q} & =\frac{\Gamma_{11}\left(\Gamma_{33} \Gamma_{24}-\Gamma_{23} \Gamma_{34}\right)+\Gamma_{12}\left(\Gamma_{13} \Gamma_{34}-\Gamma_{14} \Gamma_{33}\right)+\Gamma_{13}\left(\Gamma_{14} \Gamma_{23}-\Gamma_{13} \Gamma_{24}\right)}{\Gamma_{12}\left(\Gamma_{23} \Gamma_{34}-\Gamma_{24} \Gamma_{33}\right)+\Gamma_{13}\left(\Gamma_{23} \Gamma_{24}-\Gamma_{22} \Gamma_{34}\right)+\Gamma_{14}\left(\Gamma_{22} \Gamma_{33}-\Gamma_{23} \Gamma_{23}\right)} \\
W_{q} & =\frac{\Gamma_{11}\left(\Gamma_{22} \Gamma_{34}-\Gamma_{24} \Gamma_{23}\right)+\Gamma_{12}\left(\Gamma_{13} \Gamma_{24}-\Gamma_{12} \Gamma_{34}\right)+\Gamma_{14}\left(\Gamma_{12} \Gamma_{23}-\Gamma_{13} \Gamma_{22}\right)}{\Gamma_{12}\left(\Gamma_{23} \Gamma_{34}-\Gamma_{24} \Gamma_{33}\right)+\Gamma_{13}\left(\Gamma_{23} \Gamma_{24}-\Gamma_{22} \Gamma_{34}\right)+\Gamma_{14}\left(\Gamma_{22} \Gamma_{33}-\Gamma_{23} \Gamma_{23}\right)}(12) \\
\Phi_{q} & =\frac{\Gamma_{11}\left(\Gamma_{23} \Gamma_{23}-\Gamma_{22} \Gamma_{33}\right)+\Gamma_{12}\left(\Gamma_{12} \Gamma_{33}-\Gamma_{13} \Gamma_{23}\right)+\Gamma_{13}\left(\Gamma_{13} \Gamma_{22}-\Gamma_{12} \Gamma_{23}\right)}{\Gamma_{12}\left(\Gamma_{23} \Gamma_{34}-\Gamma_{24} \Gamma_{33}\right)+\Gamma_{13}\left(\Gamma_{23} \Gamma_{24}-\Gamma_{22} \Gamma_{34}\right)+\Gamma_{14}\left(\Gamma_{22} \Gamma_{33}-\Gamma_{23} \Gamma_{23}\right)}
\end{aligned}
$$


Using superposition and the amplitude ratios in equation (12), we finally obtain the formal solutions

$$
\left\{\begin{array}{c}
u_{1} \\
u_{2} \\
u_{3} \\
\phi \\
\bar{T}_{33} \\
\bar{T}_{13} \\
\bar{T}_{23} \\
\bar{D}_{3}
\end{array}\right\}=\left[\begin{array}{cccccccc}
1 & 1 & 1 & 1 & 1 & 1 & 1 & 1 \\
V_{1} & V_{2} & V_{3} & V_{4} & V_{5} & V_{6} & V_{7} & V_{8} \\
W_{1} & W_{2} & W_{3} & W_{4} & W_{5} & W_{6} & W_{7} & W_{8} \\
\Phi_{1} & \Phi_{2} & \Phi_{3} & \Phi_{4} & \Phi_{5} & \Phi_{6} & \Phi_{7} & \Phi_{8} \\
D_{11} & D_{12} & D_{13} & D_{14} & D_{15} & D_{16} & D_{17} & D_{18} \\
D_{21} & D_{22} & D_{23} & D_{24} & D_{25} & D_{26} & D_{27} & D_{28} \\
D_{31} & D_{32} & D_{33} & D_{34} & D_{35} & D_{36} & D_{37} & D_{38} \\
D_{41} & D_{42} & D_{43} & D_{44} & D_{45} & D_{46} & D_{47} & D_{48}
\end{array}\right]\left\{\begin{array}{c}
U_{11} E_{1} \\
U_{12} E_{2} \\
U_{13} E_{3} \\
U_{14} E_{4} \\
U_{15} E_{5} \\
U_{16} E_{6} \\
U_{17} E_{7} \\
U_{18} E_{8}
\end{array}\right\}
$$

or

$$
F=[X][D] \tilde{U}
$$

where $F$ is the interfacial components at the interface between two solid layers, $\bar{T}_{j 3}=$ $T_{j 3} / i \xi, \bar{D}_{3}=D_{3} / i \xi,[X]$ is the $8^{\circ} \times 8$ matrix of equation (13), $\tilde{U}=\left\{U_{1 q}\right\}^{\top},[D]_{k}$ is the $8 \times 8$ diagonal matrix whose entries are $E_{q}=\exp ^{i \xi \alpha_{q} x_{3}}, q=1, \ldots, 8$, and

$$
\begin{aligned}
& D_{1 q}=C_{13}+C_{36} V_{q}+C_{33} \alpha_{q} W_{q}+e_{33} \alpha_{q} \Phi_{q} \\
& D_{2 q}=C_{55} \alpha_{q}+C_{45} \alpha_{q} V_{q}+C_{55} W_{q}+e_{15} \Phi_{q} \\
& D_{3 q}=C_{45} \alpha_{q}+C_{44} \alpha_{q} V_{q}+C_{45} W_{q}+e_{14} \Phi_{q} \\
& D_{4 q}=e_{31}+e_{36} V_{q}+e_{33} \alpha_{q} W_{q}-\epsilon_{33} \alpha_{q} \Phi_{q} .
\end{aligned}
$$

With reference to the relations (11) and by inspection of equations (12)-(15) we recognize the restrictions

$$
\begin{gathered}
V_{j}=V_{j+1}, \quad W_{j}=-W_{j+1}, \quad \Phi_{j}=-\Phi_{j+1}, \\
D_{1 j}=D_{1 j+1}, D_{2 j}=-D_{2 j+1}, D_{3 j}=-D_{3 j+1}, D_{4 j}=D_{4 j+1}, j=1,3,5,7 .
\end{gathered}
$$

By eliminating the common amplitudes $\tilde{U}$, equation (14) can be used to relate the interfacial components at bottom, $\left(x_{3}^{\prime}\right)_{k}=-d_{k} / 2$, to those at top, $\left(x_{3}^{\prime}\right)_{k}=d_{k} / 2$, of the $k$ th layer. After rather lengthy algebraic reductions and manipulations we obtain (with the summation holding)

$$
F_{k}^{+}=[a]_{k} F_{k}^{-}, \quad k=1,2, \ldots, n,
$$

where

$$
[a]_{k}=[X]_{k}[D]_{k}[X]_{k}^{-1}
$$


constitutes the most general transfer matrix for the monoclinic layer $k$. It allows the wave to be incident on layer $k$ at an arbitrary angle $\theta$ from the normal $x_{3}$ or equivalently $\left(x_{3}^{\prime}\right)$ and at any azimuthal angle $\psi$. Matrix transfer for higher symmetry material such as orthotropic, transversely isotropic, and cubic can be obtained from equation (18) as asymptotically limiting cases.

The matrix transfer technique then yields, via the continuity of displacements and stresses at the various layer interfaces, i.e. $F_{k}^{+}=F_{k+1}^{-}$, the response vector at $x_{3}=d$ in terms of that at $x_{3}=0$ :

$$
F^{+}=[A] F^{-}
$$

where

$$
[A]=[a]_{n}[a]_{n-1} \ldots[a]_{1}
$$

where $[A]$ represents the global transfer matrix, and $F^{+}$and $F^{-}$the column vectors with respect to the top and bottom of the layered medium, respectively.

Waves propagating in certain crystals and along certain cuts of those crystals can be decoupled into two wave types: pure elastic waves of coupled $P$ and $S V$ waves, and piezoelectric coupled $S H$ waves [1]. The same procedure for obtaining the transfer matrix can be applied to those decoupled cases, such as in the orthotropic-222 or $-\mathrm{mm} 2$ group, respectively.

\subsection{MONOCLINIC-M CLASS}

Results for the mono-m case with the constitutive relations (8) can be obtained by following identical steps to those given above for the mono-2 case. The steps, resulting equations and the final results are similar with the exceptions of some parameter definitions as summarized below:

(i) In equation (9) $\Gamma_{14}(\alpha), \Gamma_{24}(\alpha)$, and $\Gamma_{34}(\alpha)$ are now replaced with

$$
\begin{aligned}
& \Gamma_{14}(\alpha)=e_{11}+e_{35} \alpha^{2} \\
& \Gamma_{24}(\alpha)=e_{16}+e_{34} \alpha^{2} \\
& \Gamma_{34}(\alpha)=\left(e_{13}+e_{35}\right) \alpha .
\end{aligned}
$$


(ii) The appropriate coefficients of the characteristic equation (13) are listed in Appendix $A$ of [2] under mono-m class.

(iii) Equations (15) and (16) are replaced with

$$
\begin{aligned}
& D_{1 q}=C_{13}+C_{36} V_{q}+C_{33} \alpha_{q} W_{q}+e_{13} \Phi_{q} \\
& D_{2 q}=C_{55} \alpha_{q}+C_{45} \alpha_{q} V_{q}+C_{55} W_{q}+e_{35} \Phi_{q} \\
& D_{3 q}=C_{45} \alpha_{q}+C_{44} \alpha_{q} V_{q}+C_{45} W_{q}+e_{34} \Phi_{q} \\
& D_{4 q}=e_{35} \alpha_{q}+e_{34} \alpha_{q} V_{q}+e_{35} W_{q}-\epsilon_{33} \alpha_{q} \Phi_{q}
\end{aligned}
$$

and

$$
\begin{gathered}
V_{j}=V_{j+1}, \quad W_{j}=-W_{j+1}, \quad \Phi_{j}=\Phi_{j+1}, \\
D_{1 j}=D_{1 j+1}, \quad D_{2 j}=-D_{2 j+1}, \quad D_{3 j}=-D_{3 j+1}, \quad D_{4 j}=-D_{4 j+1} .
\end{gathered}
$$

\subsection{TOP FLUID BOUNDARY}

The multilayered medium is bounded with a fluid half-space on its top surface and a substrate on its bottom. The input wave is assumed to be periodic and originating in the top fluid half-space and incident on the medium at an arbitrary angle from the normal. The displacements and stresses within the top fluid are given by properly specializing equation (13) and recognizing the absence of shear deformation and electric potential within the fluid so that

$$
\left\{\begin{array}{c}
u_{1}^{f} \\
u_{3}^{f} \\
\bar{T}_{33}^{f}
\end{array}\right\}=\left[\begin{array}{cc}
1 & 1 \\
\alpha_{f} & -\alpha_{f} \\
\rho_{f} c^{2} & -\rho_{f} c^{2}
\end{array}\right]\left\{\begin{array}{c}
U_{1}^{f} \exp ^{i \xi \alpha_{f}\left(x_{3}-d\right)} \\
U_{2}^{f} \exp ^{-i \xi \alpha_{f}\left(x_{3}-d\right)}
\end{array}\right\} \exp ^{i \xi\left(x_{1}-c t\right)}
$$

where $\bar{T}_{33}^{f}=T_{33}^{f} / i \xi, \alpha_{f}^{2}=\left(c^{2} / c_{f}^{2}\right)-1, U_{1}^{f}$ is the constant amplitude of the incoming wave, $U_{2}^{f}$ is that of the reflected wave and the sub and superscripts $f$ denote quantities belonging to the fluid. The continuity conditions at the plate-fluid interface are given by

$$
u_{2}^{f}=u_{2}^{n}, \quad T_{33}^{f}=T_{33}^{n}, \quad \phi^{n}=T_{13}^{n}=T_{23}^{n}=0, \quad \text { at } x_{3}=d .
$$




\subsection{BOTTOM BOUNDARY SOLID SUBSTRATE}

Since the bottom bounding medium is a solid substrate, its response is given by specializing equation (13) to the substrate material and again ensuring boundness of the solution by selecting only the appropriate amplitudes within the substrate so that the substrate amplitude vector is

$$
\left\{U_{1}^{s}, 0, U_{3}^{s}, 0, U_{5}^{s}, 0, U_{7}^{s}, 0\right\}^{\top}
$$

The appropriate conditions at the bottom plate-substrate interface require the continuity of all displacements, stress components, and electric potential. Satisfying such conditions for a shorted solid substrate finally yields the reflection coefficient

$$
R=\frac{U_{2}^{f}}{U_{1}^{f}}=\frac{M_{1}-Q_{f} M_{2}}{M_{1}+Q_{f} M_{2}}
$$

and the quasilongitudinal, quasitransverse shear and electric potential transmission coefficients

$$
\begin{aligned}
T_{L} & =\frac{U_{1}^{s}}{U_{1}^{f}}=\frac{2 Q_{f} P_{1}}{M_{1}+Q_{f} M_{2}}, \\
T_{3}^{s}=\frac{U_{3}^{s}}{U_{1}^{f}}=\frac{-P_{3}}{P_{1}} T_{L}, \quad T_{5}^{s} & =\frac{U_{5}^{s}}{U_{1}^{f}}=\frac{-P_{5}}{P_{1}} T_{L}, \quad T_{7}^{s}=\frac{U_{7}^{s}}{U_{1}^{f}}=\frac{-P_{7}}{P_{1}} T_{L}
\end{aligned}
$$

where $Q_{f}=\rho_{f} c^{2} / \alpha_{f}$

$$
\begin{array}{rlrl}
M_{1} & =\left|\begin{array}{llll}
P_{51} & P_{53} & P_{55} & P_{57} \\
P_{41} & P_{43} & P_{45} & P_{47} \\
P_{61} & P_{63} & P_{65} & P_{67} \\
P_{71} & P_{73} & P_{75} & P_{77}
\end{array}\right| & M_{2}=\left|\begin{array}{llll}
P_{31} & P_{33} & P_{35} & P_{37} \\
P_{41} & P_{43} & P_{45} & P_{47} \\
P_{61} & P_{63} & P_{65} & P_{67} \\
P_{71} & P_{73} & P_{75} & P_{77}
\end{array}\right| \\
P_{1}=\left|\begin{array}{lll}
P_{43} & P_{45} & P_{47} \\
P_{63} & P_{65} & P_{67} \\
P_{73} & P_{75} & P_{77}
\end{array}\right| & P_{3}=\left|\begin{array}{llll}
P_{41} & P_{45} & P_{47} \\
P_{61} & P_{65} & P_{67} \\
P_{71} & P_{75} & P_{77}
\end{array}\right| \\
P_{5}=\left|\begin{array}{lll}
P_{41} & P_{43} & P_{47} \\
P_{61} & P_{63} & P_{67} \\
P_{71} & P_{73} & P_{77}
\end{array}\right| & P_{7}=\left|\begin{array}{lll}
P_{41} & P_{43} & P_{45} \\
P_{61} & P_{63} & P_{65} \\
P_{71} & P_{73} & P_{75}
\end{array}\right|
\end{array}
$$

and $\left[P_{i j}\right]$ is the matrix product of $\left[A_{i q}\right]$ from equation (20) and the substrate's characteristic matrix from the specialization of equation (13) to the substrate. 


\section{QUALITATIVE DESCRIPTION OF RESULTS}

So far we have derived expressions for the reflection and transmission coefficients, shown in equation (27), for multilayered monoclinic substrates with piezoelectric in.teraction. Results for arbitrary orientations of the individual layers, arbitrary frequencies and angles of incidence are readily available. The reflection and transmission coefficients contain, as a by-product, the characteristic equation for the propagation of leaky surface waves on the corresponding medium. The vanishing of the denominator in equation (27), namely,

$$
M_{1}+Q_{f} M_{2}=0
$$

defines the characteristic equation for the propagation of leaky surface waves on the multilayered substrate. In the absence of the fluid equation (29) reduces to

$$
M_{1}=0
$$

defining the characteristic equation for the dry system.

The similar results for free case can be obtained by assuming that there is a very thin insulated coating on the surface of the top layer and setting the electric displacement $D_{3}$, rather than the electric potential, to vanish. The free case will not be demonstrated here and can be derived without any problem by following the same procedure shown for the shorted case.

The fraction change of phase velocities $\Delta v / v$, so-called the piezoelectric coupling factor, is used to evaluate the coupling efficiency and is defined as

$$
\frac{\Delta v}{v}=\frac{v_{\infty}-v_{0}}{v_{\infty}}
$$

where $v_{\infty}$ and $v_{0}$ are the phase velocities of free and shorted cases, respectively.

\section{NUMERICAL ILLUSTRATIONS AND DIS- CUSSION}

It is known that the Rayleigh wave propagating in a single substrate is not dispersive with respect to frequency but to the propagation direction. However, wave prop- 


\begin{tabular}{||c|c|c|c||}
\hline \hline $\begin{array}{c}\text { IFaterial } \\
\text { Properties }\end{array}$ & $\begin{array}{c}\text { Quartz } \\
\text { Trig.-3m }\end{array}$ & $\begin{array}{c}\text { GaAs } \\
\text { Cubic-43m }\end{array}$ & $\begin{array}{c}\text { PZT-65/35 } \\
\text { Uniaxial }\end{array}$ \\
\hline$\rho$ & 2.651 & 5.307 & 7.500 \\
\hline \hline$C_{11}$ & 86.74 & 118.8 & 159.4 \\
\hline$C_{33}$ & 107.20 & 118.8 & 126.1 \\
\hline$C_{44}$ & 57.94 & 59.4 & 38.9 \\
\hline$C_{66}$ & 39.87 & 59.4 & 42.8 \\
\hline$C_{12}$ & 6.99 & 53.8 & 73.9 \\
\hline$C_{13}$ & 11.91 & 53.8 & 73.9 \\
\hline$C_{14}$ & -17.91 & & \\
\hline \hline$e_{11}$ & 0.171 & & \\
\hline$e_{14}$ & -0.0436 & 0.154 & \\
\hline$e_{15}$ & & & 8.39 \\
\hline$e_{31}$ & & & -6.13 \\
\hline$e_{33}$ & & & 10.70 \\
\hline \hline$\epsilon_{11}$ & 4.52 & 12.5 & 639.3 \\
\hline$\epsilon_{33}$ & 4.68 & 12.5 & 153.3 \\
\hline \hline \multicolumn{4}{|l}{} \\
\hline \hline
\end{tabular}

Table 1: Material properties of selected piezoelectric materials. Units of $C_{p q}, e_{i p}$, and $\rho$ are $10^{9} \mathrm{Nt} / \mathrm{m}^{2}, 10^{9} \mathrm{Coulomb} / \mathrm{m}^{2}$, and $\mathrm{g} / \mathrm{cm}^{3}$, respectively. $\epsilon_{i j}$ is given undimensional as $\epsilon^{s} / \epsilon_{0}^{s}$, where $\epsilon_{0}^{s}=8.854 \times 10^{-12} \mathrm{farad} / \mathrm{m}$.

agating in a multilayered substrate is a function of the propagation direction, the frequency, and the thickness of each layer. We have derived the analytical expressions of the reflection and transmission coefficients, equation (27), and the associated characteristic equations, equation (29), for this problem. Numerical results are presented below in two categories as the following: in the first, the reflection coefficients and the function $M_{1}$ under various phase velocity $c$ (or equivalently with incident angle $\theta \operatorname{since} \sin \theta=c_{f}(c)$ and azimuthal angles $\psi$ are illustrated. This will display the criteria of surface mode identification. The dispersion relations in the form of variations of phase velocities with $F d$ of a $G a A s$ layer on a $x_{1}$-cut Quartz substrate are illustrated and discussed. Then, the dispersion curves of multilayered substrate with different number of layers, orientations, and lamination orders of $P Z T-65 / 35$ and GaAs are presented. The material properties of the selected piezoelectric materials are listed in Table 1. 
Fig. 2 shows that the behavior of the reflection coefficient, which is a function of frequency and the thickness of each layer, for a multilayered substrate. Analysis of the behavior of the reflection coefficient allows us to identify all of the propagation characteristics which influence the distribution of the reflected field. Fig. 2a-d show the variations of the real and imaginary parts of the reflection coefficients with phase velocity at four different $F d$ for a $G a A s$ plate with $\psi=30^{\circ}$ rigidly bonded on a $x_{1}$-cut Quartz substrate. Also displayed in these figures are the normalized values of the corresponding parameters $M_{1}$. The wave behavior in the presence of liquid (socalled leaky Rayleigh surface wave) [1] is also applied here, i.e. where the real part of the reflection coefficient approaches -1 which also coincides with the rapid variation (through zero) of its phase and the vanishing of the function $M_{1}$. Furthermore, at $F d=0$, only one mode occurs and is at the surface wave speed of the substrate which is $3.264 \mathrm{~km} / \mathrm{sec}$ for the $x_{1}$-cut Quartz substrate. This is expected since at the zero frequency limit, i.e., for very long wavelengths, the plate will be essentially "washed" out. As the frequency increases more modes appear successively; this behavior is typical of all softening (loading) materials. By using the criteria stated previously and collecting the modes for various $F d$, a dispersion curve for the shorted case of this case is obtained and illustrated in Fig. 3. The fundamental mode, as discussed above, starts from the surface wave velocity of the $x_{1}$-cut Quartz substrate. The bulk shear velocity of the substrate is the cut-off phase velocity for higher modes and these modes do not exist if the phase velocity is higher than that velocity. Therefore, higher modes start from the so-called cut-off frequencies. For high $F d$ the fundamental mode approaches the surface wave velocity, $2.68 \mathrm{~km} / \mathrm{sec}$, of the GaAs.

In the following demonstrations, the multilayered media are chosen by putting either GaAs or PZT-65/35 as the substrate and the other as the top layer with different number of laminations of them on the substrate, where each layer has equal thickness. The influence of the layer ordering in wave characteristics is also implicitly included in this series of demonstrations. Fig. 4 shows the dispersion curve for a $P Z T-65 / 35$ layer on a $G a A s$ substrate with $\psi=45^{\circ}$, where solid and dashed lines 
are for shorted and free cases, respectively. Because the medium is softened by the $P Z T-65 / 35$ layer, the phase velocity starts from the surface wave velocity of the substrate $(2.87 \mathrm{~km} / \mathrm{sec})$ at $f d=0$ and approaches to that of the layer $(2.38 \mathrm{~km} / \mathrm{sec})$ at high $f d$. Higher modes exist and have cut-off velocities at the bulk shear velocity of the substrate $(3.34 \mathrm{~km} / \mathrm{sec})$. By selecting a $G a A$ s layer on a $P Z T-65 / 35$ substrate, there exists only one mode because the layer is stiffer than the substrate. This leaky type of wave, starting from the surface velocity of the substrate $(2.38 \mathrm{~km} / \mathrm{sec})$ and being cut-off at that of the layer $(2.62 \mathrm{~km} / \mathrm{sec})$, is shown in Fig. 5 .

Fig. 6 shows the dispersion curve for GaAs and PZT-65/35 layers on a $G a A s$ substrate with $\psi=45^{\circ}$. The fundamental mode starts at $2.87 \mathrm{~km} / \mathrm{sec}$ and decreases if $f d$ increases, like the case in Fig. 4 , because of the softening. However, if the frequency keeps on increasing, the phase velocity will decrease because of the extra layer of $G a A s$ which stiffen the medium at high $f d$. By adding an addition layer of PZT$65 / 35$ on top of the case shown in Fig. 5 , higher modes exist rather than just a single fundamental mode. Fig. 7 shows that the phase velocity of the fundamental mode starts from $2.38 \mathrm{~km} / \mathrm{sec}$, increases if $f d$ increased, and will decrease and approach to $2.38 \mathrm{~km} / \mathrm{sec}$ if $f d$ keeps on increasing. Figs. 8 and 9 illustrate the piezoelectric coupling factor for the last two cases, GaAs and PZT-65/35 layers on GaAs substrate and $P Z T-65 / 35$ and $G a A s$ on $P Z T-65 / 35$ substrate, respectively.

Fig. 10 show that four GaAs and PZT-65/63 laminations on a GaAs substrate, while the Fig. 11 illustrates a $P Z T-65 / 63$ substrate with four PZT-65/63 and $G a A s$ laminations. Both cases exhibits similar behavior as the last two cases, respectively. However, with the increasing of the layer number, the phase velocity of the fundamental mode has minor pertubation and the cut-off frequencies of higher modes are increased.

\section{CONCLUSION}

A theoretical investigations and formulations for ultrasonic waves in the multilayered piezoelectric substrates with fluid loading are presented. Each layer processes up to as 
low as monoclinic symmetry. Reflection and transmission coefficients are derived from which all characteristic behavior of the system is identified. Solutions are obtained for the individual layers which relate the field variables at the upper and lower layer surfaces and by satisfying appropriate interfacial conditions across the layers. It was also proved that the results obtained under this assumption from fluid-loaded cases are same as that of dry cases. Our results are rather general and contain a wide variety of special cases. Multilayered substrates with different orientations, number of layers, materials, and lamination order can be studied. Some numerical results for multilayered piezoelectric substrates are demonstrated.

\section{DISCLAIMER}

This report was prepared as an account of work sponsored by an agency of the United States Government. Neither the United States Government nor any agency thereof, nor any of their employees, makes any warranty, express or implied, or assumes any legal liability or responsibility for the accuracy, completeness, or usefulness of any information, apparatus, product, or process disclosed, or represents that its use would not infringe privately owned rights. Reference herein to any specific commercial product, process, or service by trade name, trademark, manufacturer, or otherwise does not necessarily constitute or imply its endorsement, recommendation, or favoring by the United States Government or any agency thereof. The views and opinions of authors expressed herein do not necessarily state or reflect those of the United States Government or any agency thereof. 


\section{REFERENCES}

[1] A. H. Nayfeh and H.-T. Chien, "The Influence of Piezoelectricity on Free and Reflected Waves From Fluid Loaded Anisotropic Plates," Journal of Acoustical Society of America, vol. 91, pp. 1250-1261, 1992.

[2] A. H. Nayfeh and H.-T. Chien, "The Influence of Piezoelectricity on Free and Reflected Waves From Fluid Loaded Anisotropic Substrates," Journal of Acoustical Society of America, vol. 91, pp. 3126-3135, 1992.

[3] E. G. Henneke II, "Reflection-Refraction of a Stress Wave at a Plane Boundary between Anisotropic Media," Journal of Acoustical Society in America, vol. 51, pp. 210-217, 1972.

[4] A. H. Nayfeh, "Elastic Wave Reflection from Liquid-anisotropic Substrate Interfaces," Wave Motion, vol. 14, pp. 55-67, 1991.

[5] T. C. Lim and G. W. Farnell, "Character of Pseudo Surface Waves on Anisotropic Crystals," Journal of Acoustical Society in America, vol. 45, pp. 845-851, 1969.

[6] A. H. Nayfeh, "The General Problem of Elastic Wave Propagation in Multilayered Anisotropic Media," Journal of Acoustical Society in America, vol. 89, pp. 1521-1531, 1991.

[7] A. H. Nayfeh and D. E. Chimenti, "Ultrasonic Wave Reflection From LiquidLoaded Orthotropic Plates with Application to Fibrous Composites," Journal of Applied Mechanics, Transaction of ASME, vol. 55, pp. 863-868, 1988.

[8] _Theoretical Wave Propagation in Multilayered Orthotropic Media," in A. K. Mal and T. C. T. Ting, Ed., Wave Propagation in Structural Composites, vol. 90, New York: ASME, 1988, pp. 17-28.

[9] H. F. Tiersten, Linear Piezoelectric Plate Vibrations, New York: Plenum, 1969.

[10] B. A. Auld, Acoustic Fields and Waves in Solids, vol. I\&II. New York: Wiley, 1973. 


\section{LIST OF FIGURES}

Figure 1: Geometry and modeling of a multilayered medium. Incident beam strikes medium at angle $\theta$ between the principle axis of material crystal and the plane of incidence.

Figure 2: Variation of reflection coefficient with phase velocity for various frequency of a GaAs layer on a $x_{1}$-cut Quartz substrate. Solid line is the real part, long dashed line is the imaginary part, and the short dashed line is the absolute value of secular equation. (2a: $F d=0 ; 2 \mathrm{~b}: F d=0.5 ; 2 \mathrm{c}: F d=1 ; 2 \mathrm{~d}: F d=3$ )

Figure 3: Dispersion plots for a $G a A s$ layer on a $x_{1}$-cut Quartz substrate for shorted case collected from Fig. 2.

Figure 4: Dispersion plot for a PZT-65/35 layer on GaAs substrate with $\psi=45^{\circ}$. Solid and dashed lines are for shorted and free cases, respectively.

Figure 5: Dispersion plot for a $G a A s$ layer with $\psi=45^{\circ}$ on $P Z T-65 / 35$ substrate. Solid and dashed lines are for shorted and free cases, respectively.

Figure 6: Dispersion plot for GaAs and PZT-65/35 layers on $G a A s$ substrate with $\psi=45^{\circ}$. Solid and dashed lines are for shorted and free cases, respectively.

Figure 7: Dispersion plot for $P Z T-65 / 35$ and $G a A s$, with $\psi=45^{\circ}$, layers on $P Z T$ $65 / 35$ substrate. Solid and dashed lines are for shorted and free cases, respectively.

Figure S: Variation of piezoelectric coupling constant of first two modes with $F d$ for $G a A s$ and $P Z T-65 / 35$ layers on $G a A s$ substrate.

Figure 9: Variation of piezoelectric coupling constant of first two modes with $F d$ for $P Z T-65 / 35$ and $G a A s$ layers on $P Z T-65 / 35$ substrate.

Figure 10: Dispersion plot for four GaAs and PZT-65/35 laminations on GaAs substrate with $\psi=45^{\circ}$. Solid and dashed lines are for shorted and free cases, respectively.

Figure 11: Dispersion plot for four PZT-65/35 and GaAs laminations on PZT-65/3.5 substrate. Solid and dashed lines are for shorted and free cases, respectively. 


\section{LIST OF TABLES}

Table 1: Material properties of selected piezoelectric materials. Units of $C_{p q}, e_{i p}$, and $\rho$ are $10^{9} \mathrm{Nt} / \mathrm{m}^{2}, 10^{9}$ Coulomb $/ \mathrm{m}^{2}$, and $\mathrm{g} / \mathrm{cm}^{3}$, respectively. $\epsilon_{i j}$ is given undimensional as $\epsilon^{s} / \epsilon_{0}^{s}$, where $\epsilon_{0}^{s}=8.854 \times 10^{-12} \mathrm{farad} / \mathrm{m}$. 


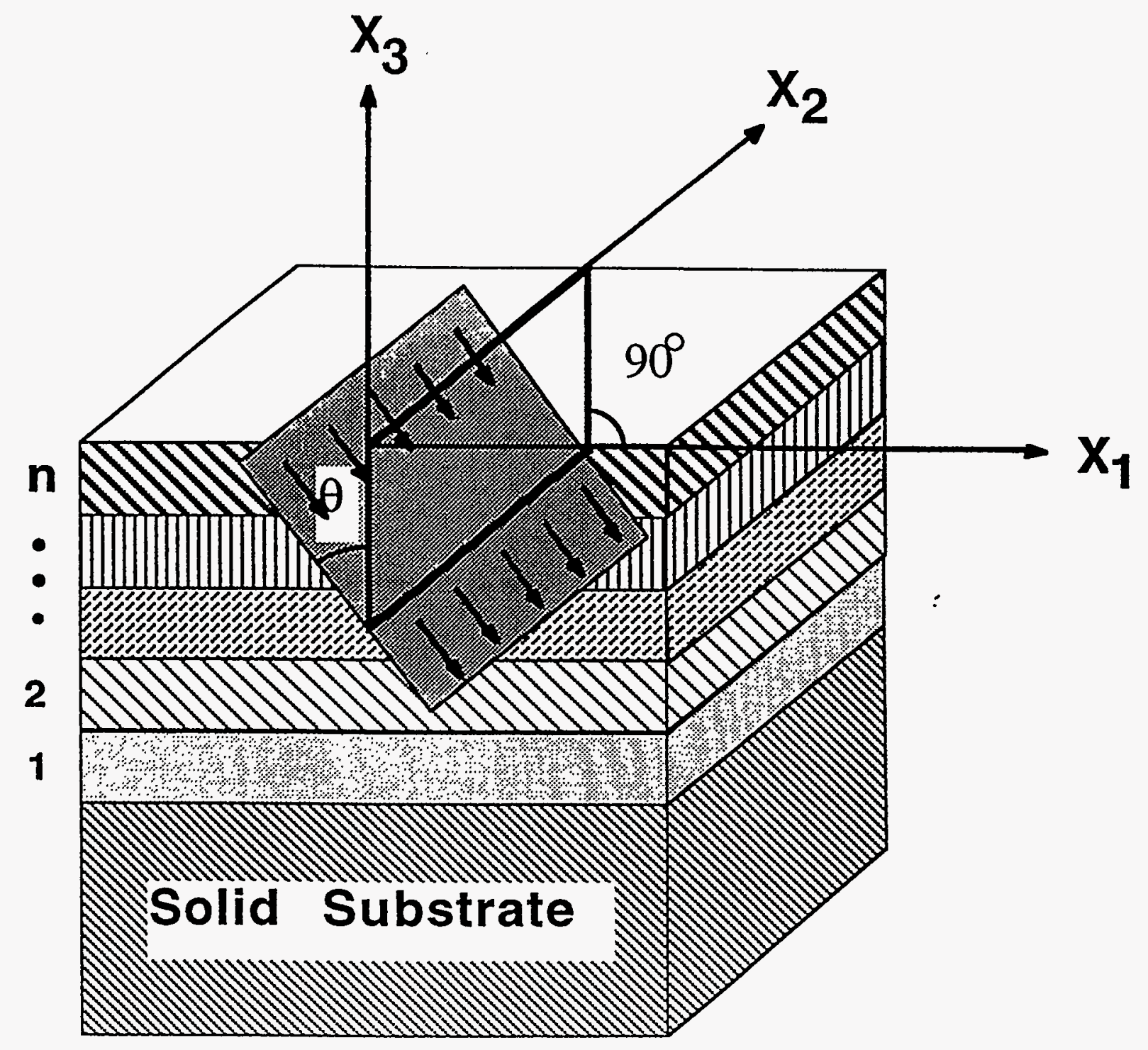


ن
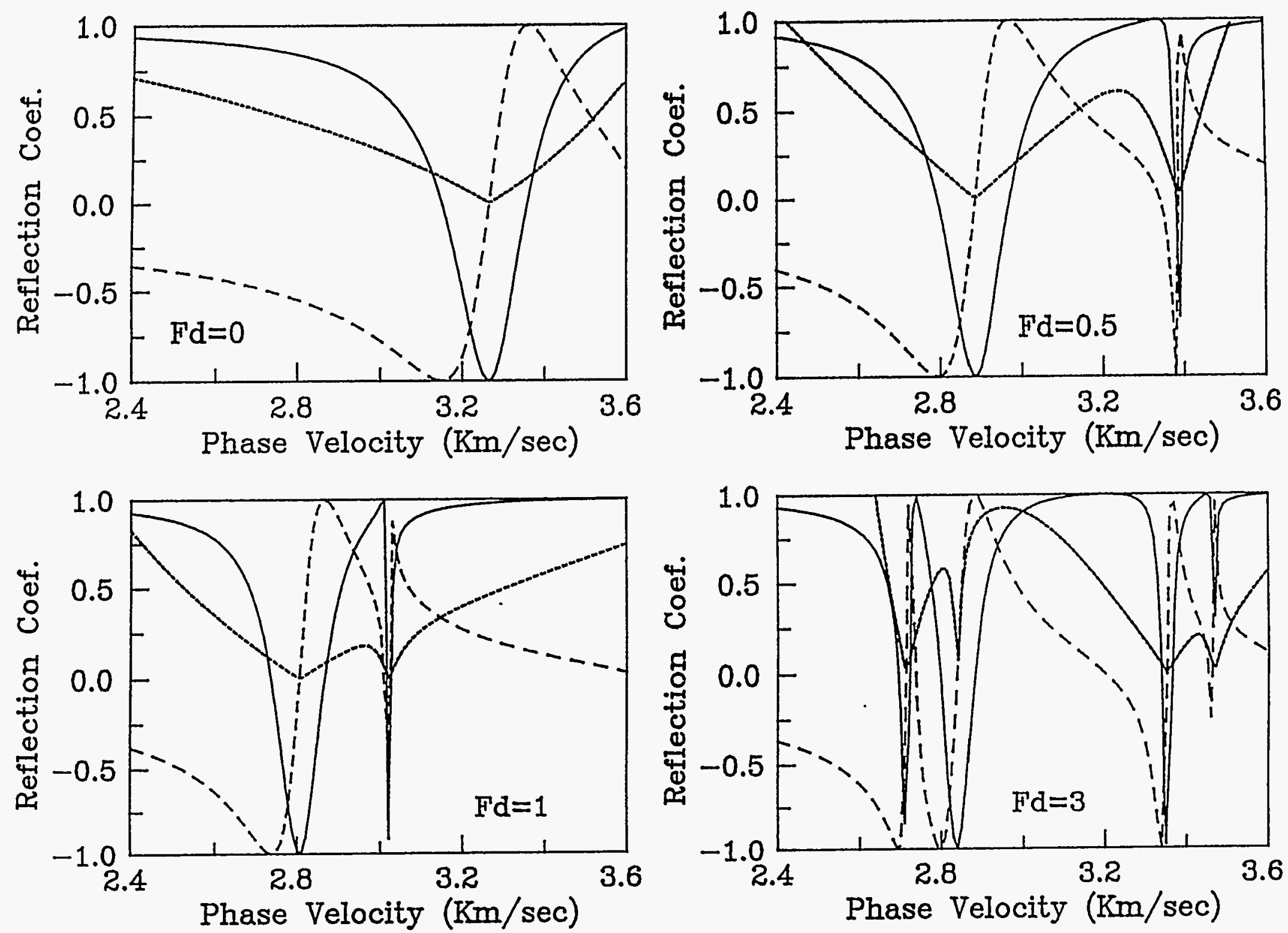
Fig. 3.

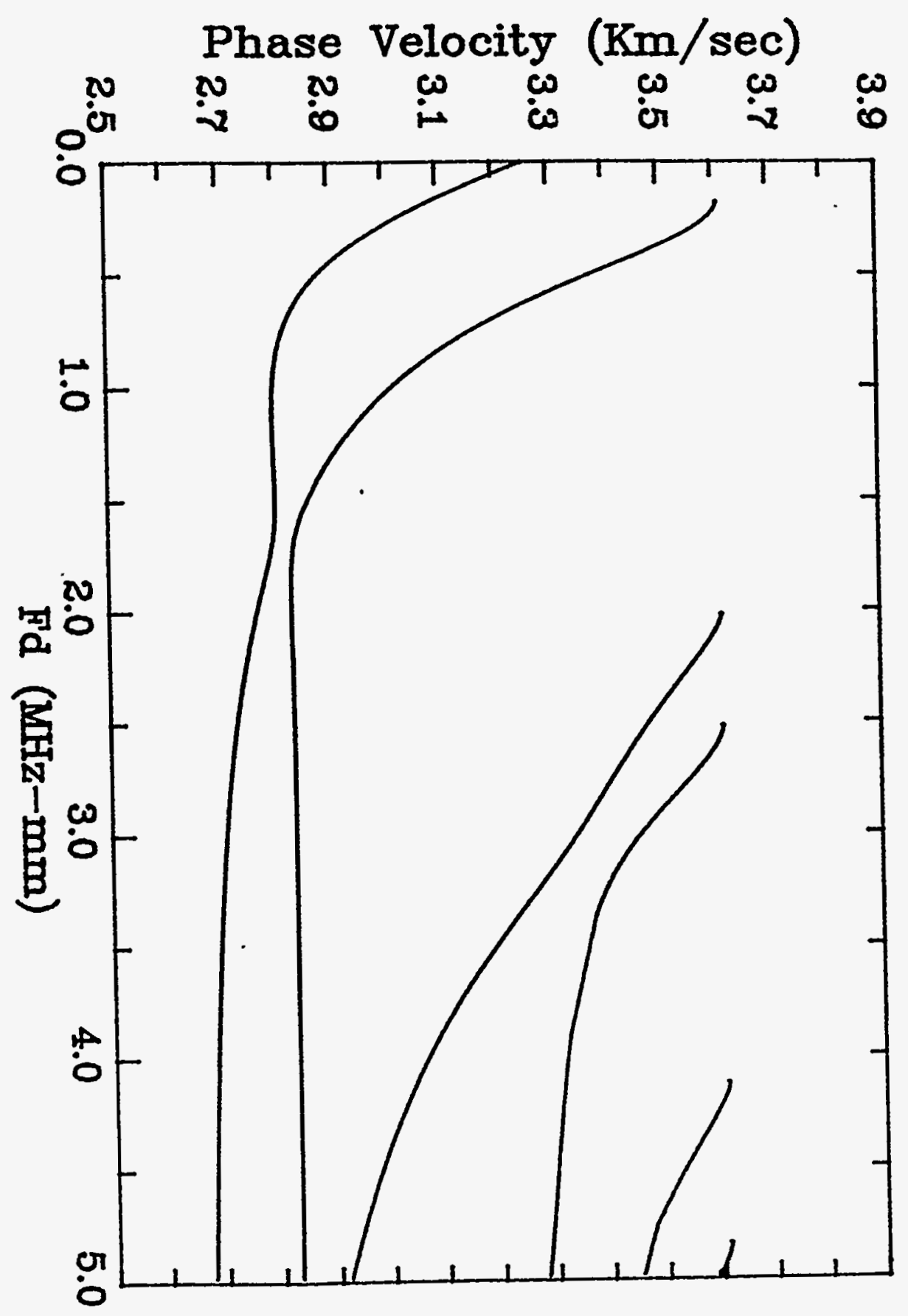


Fig 4.

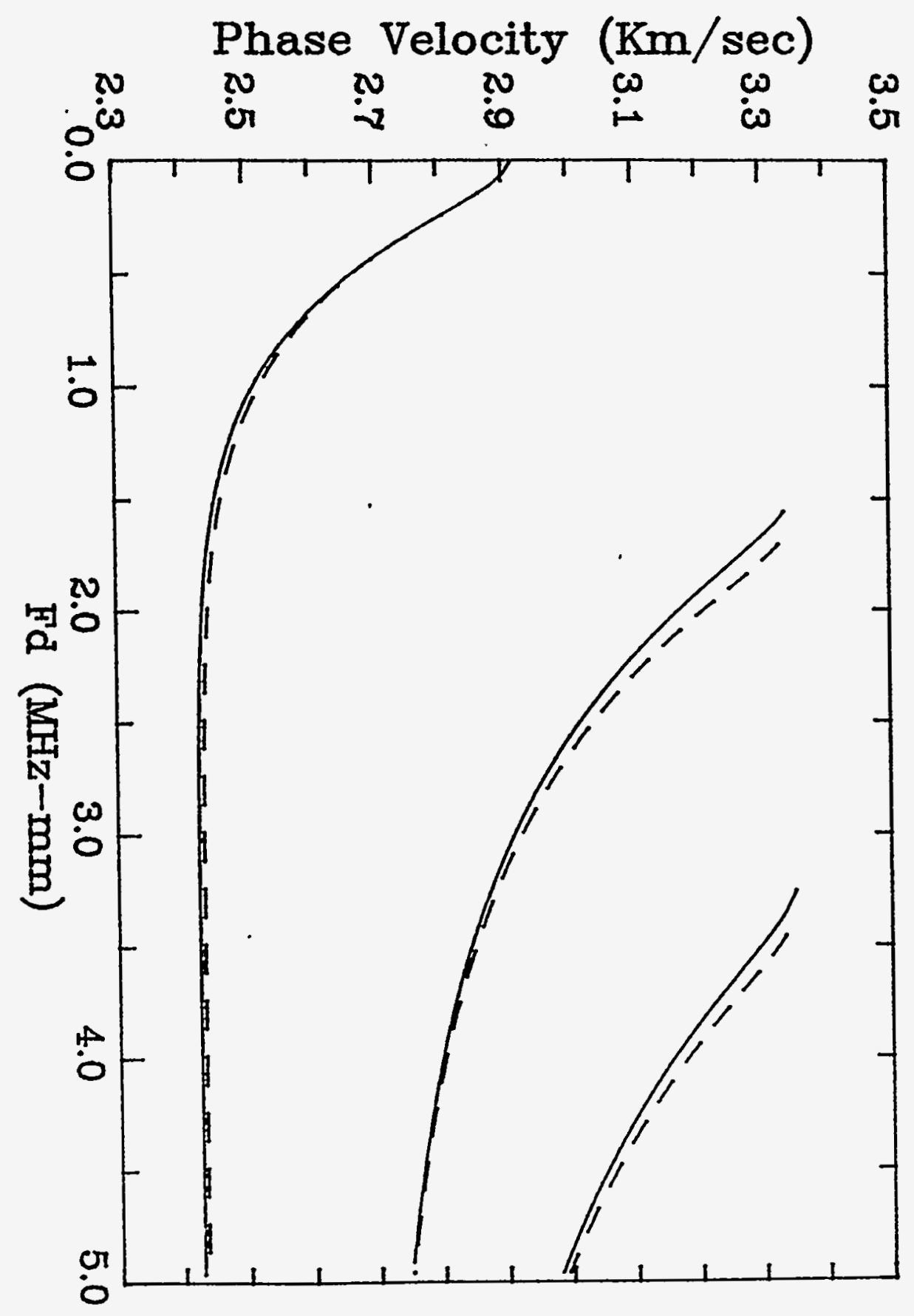


Fig. 5.

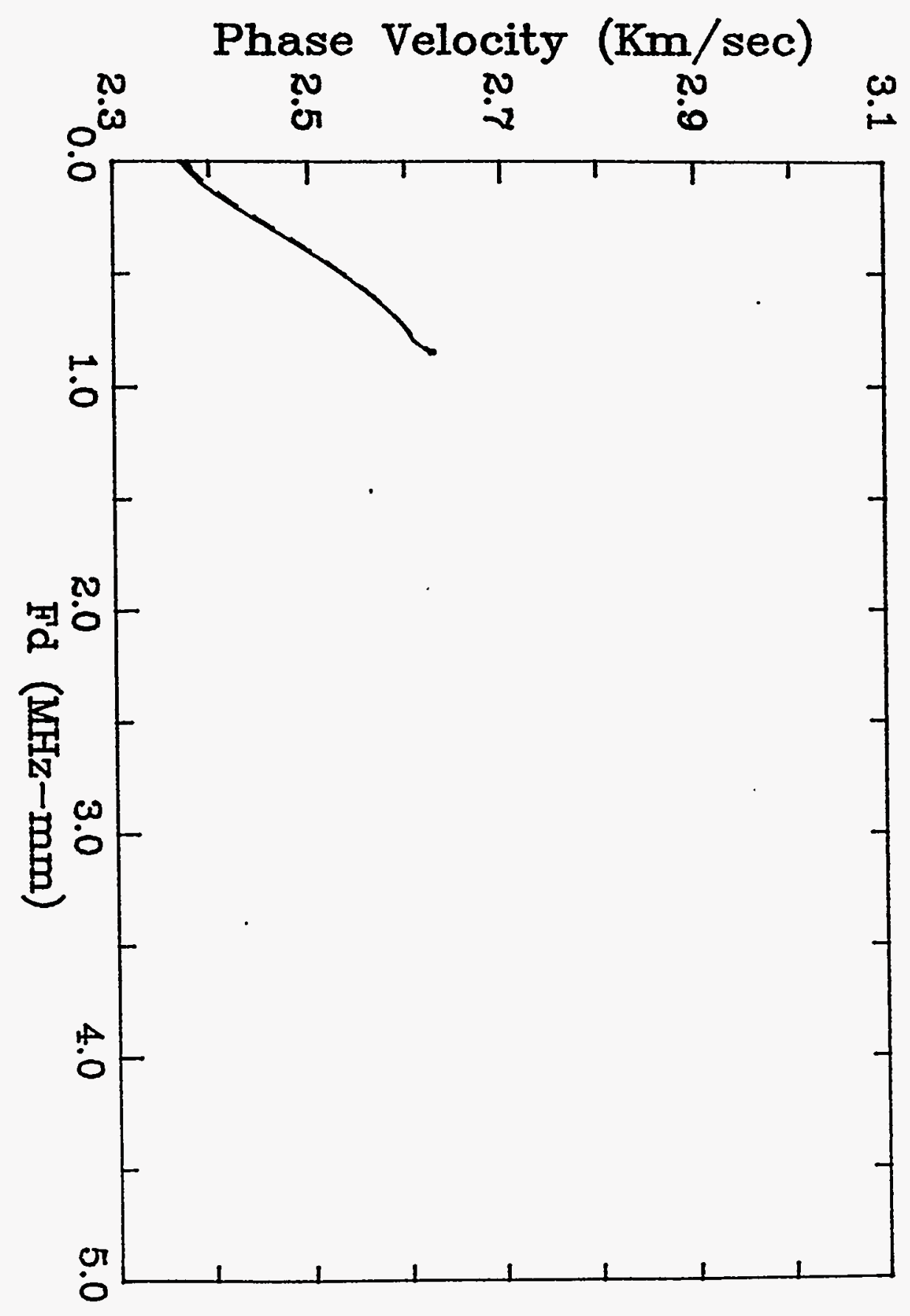


$\because \quad$

Fig. 6.

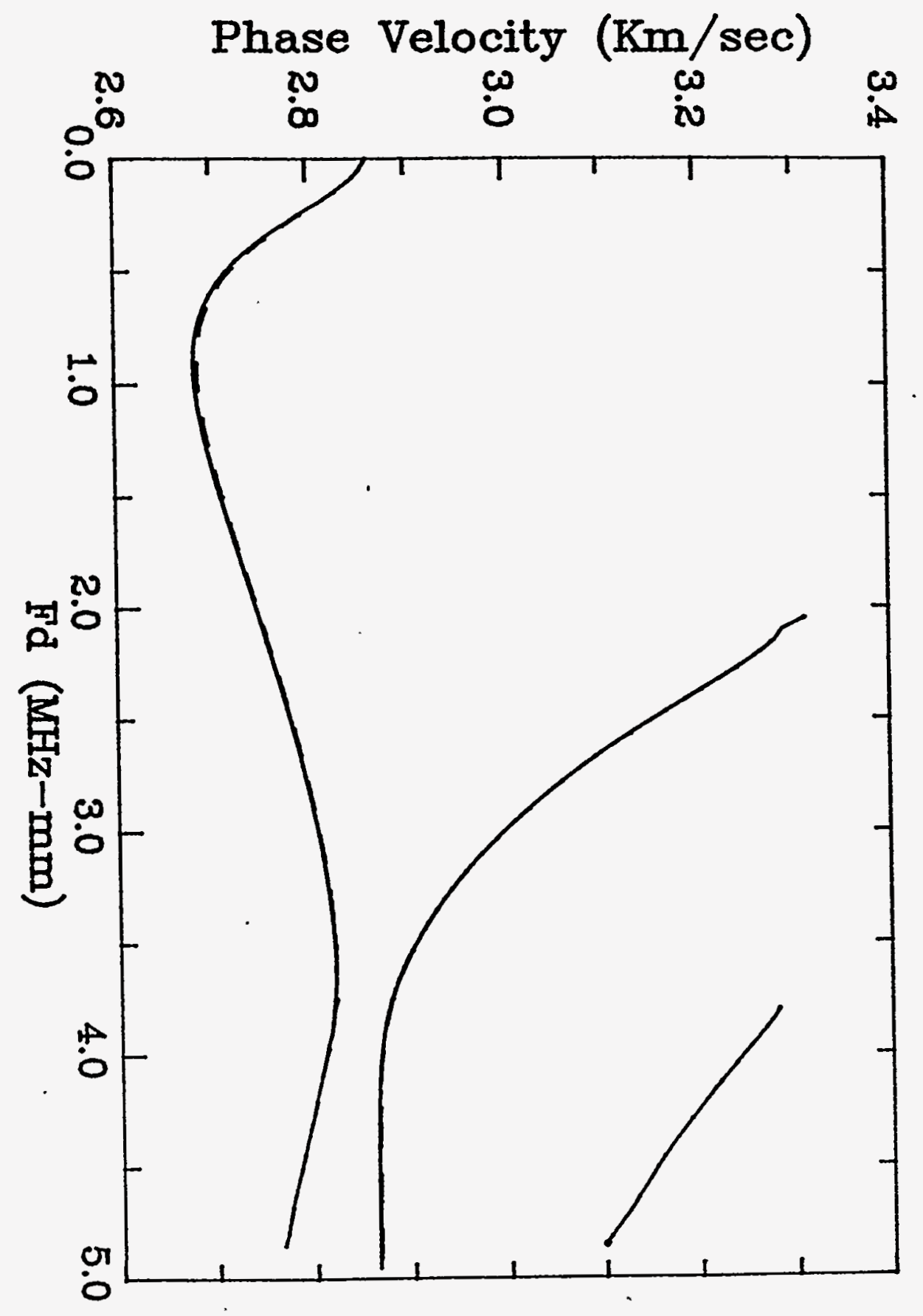




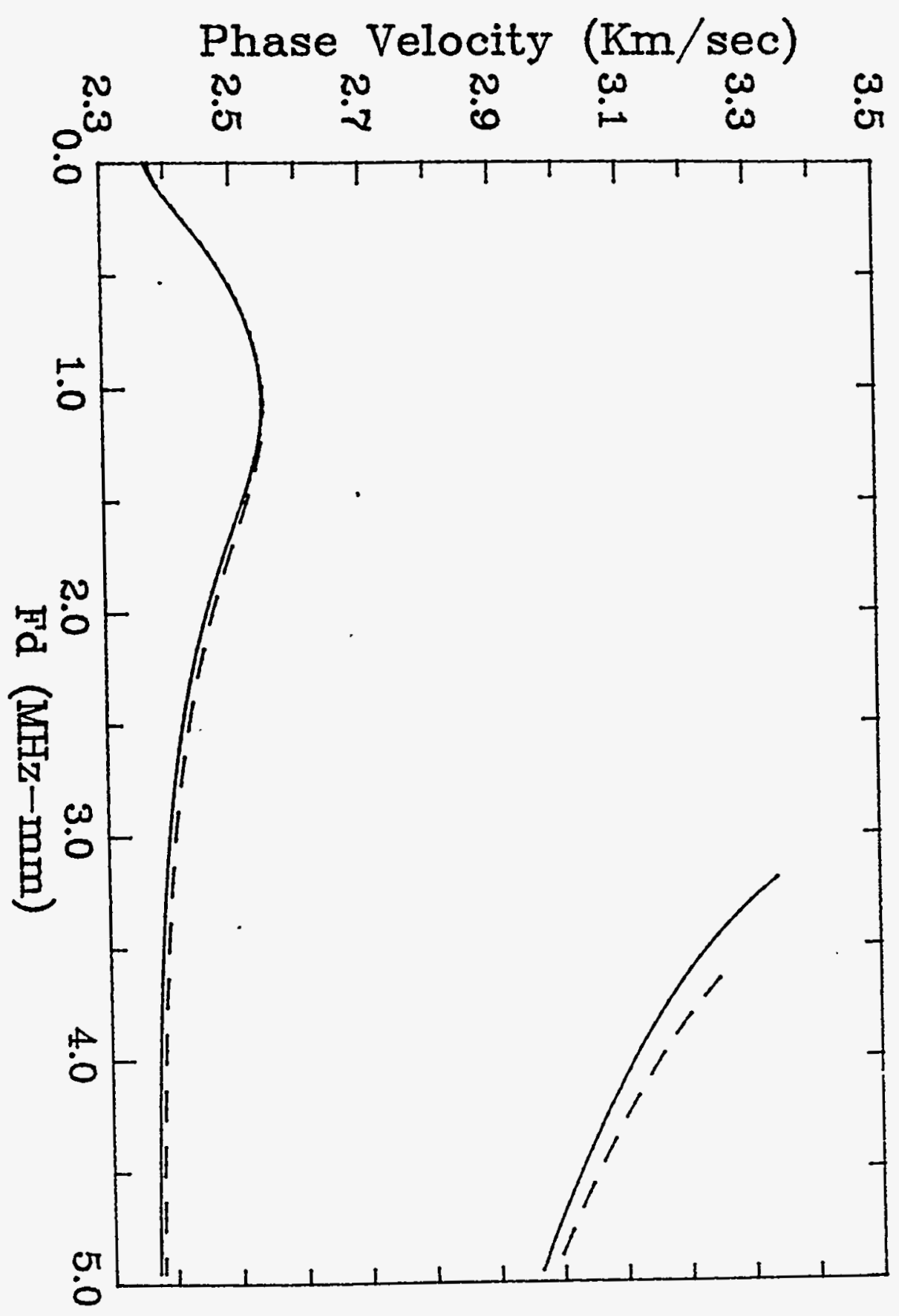


Fig. 8

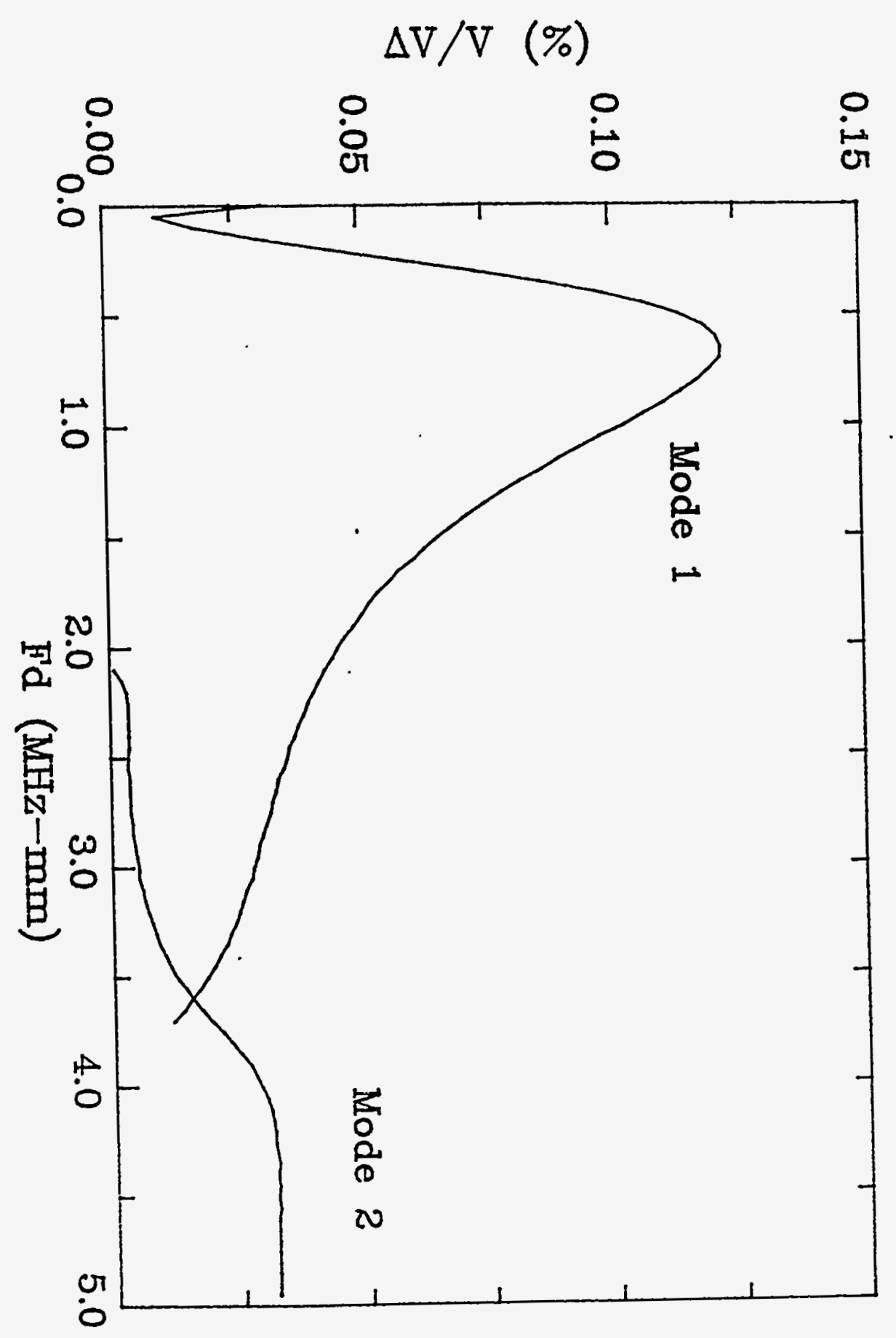


Fig. 9.

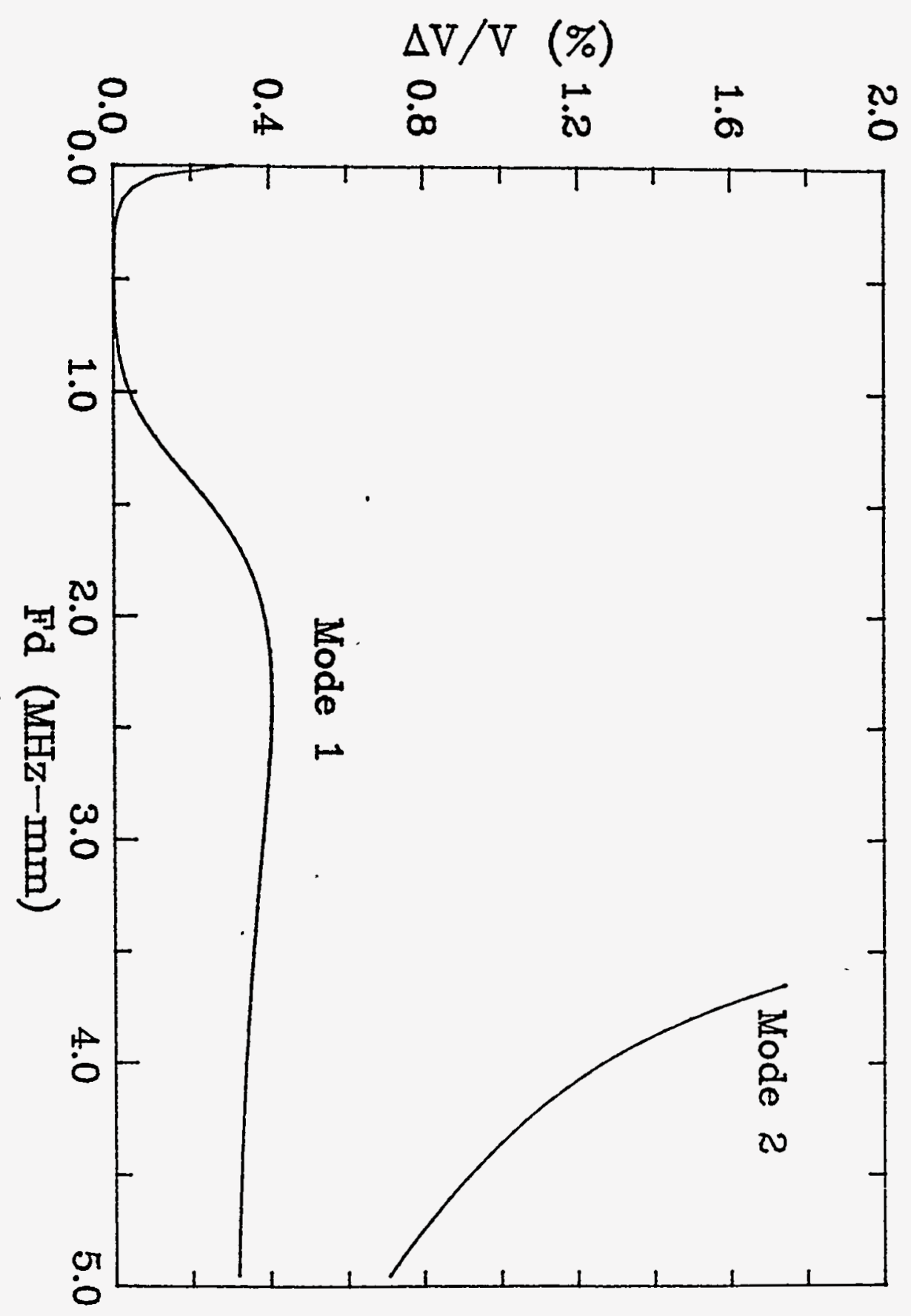


Fig. 10.

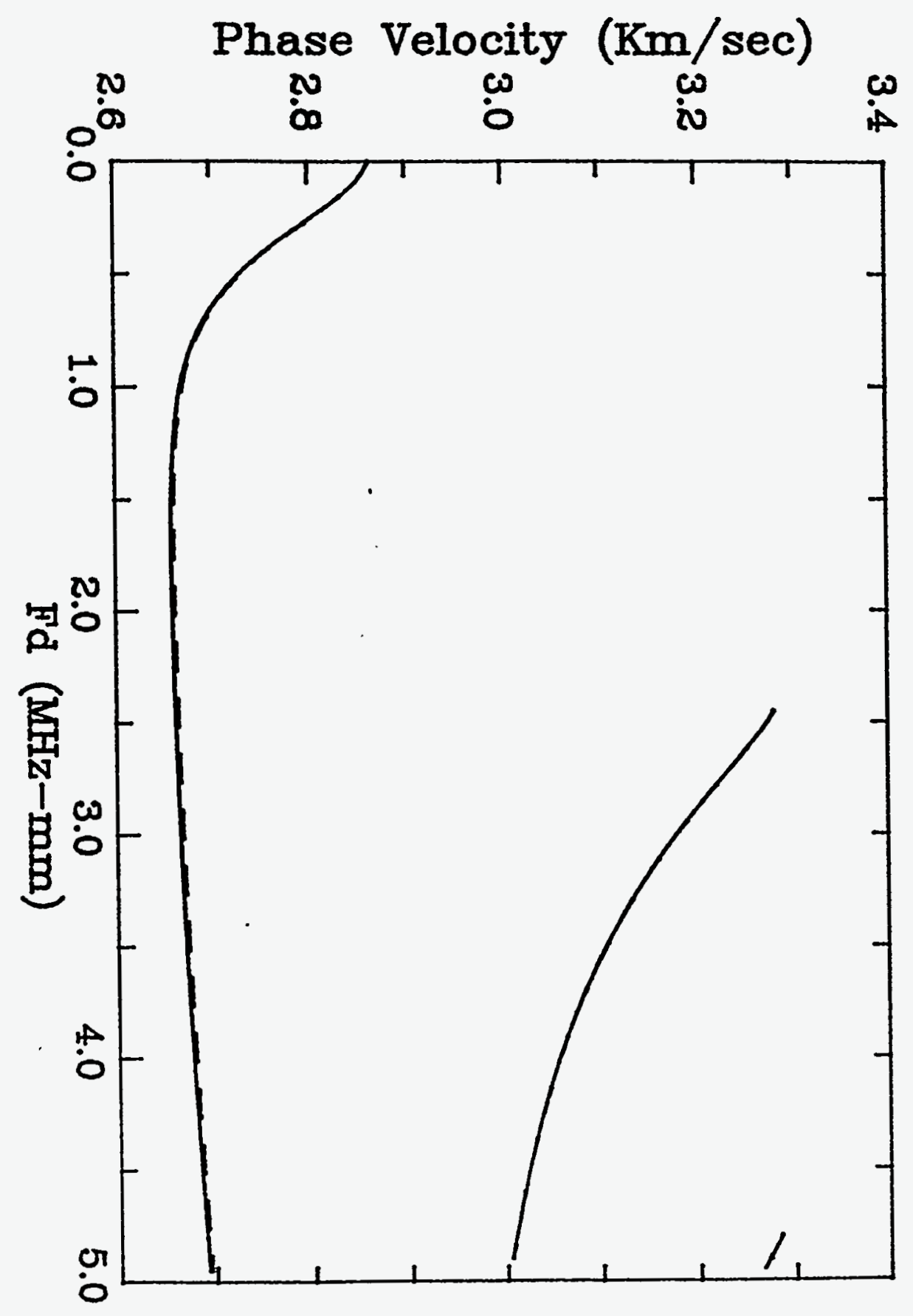


Frg. 11 .

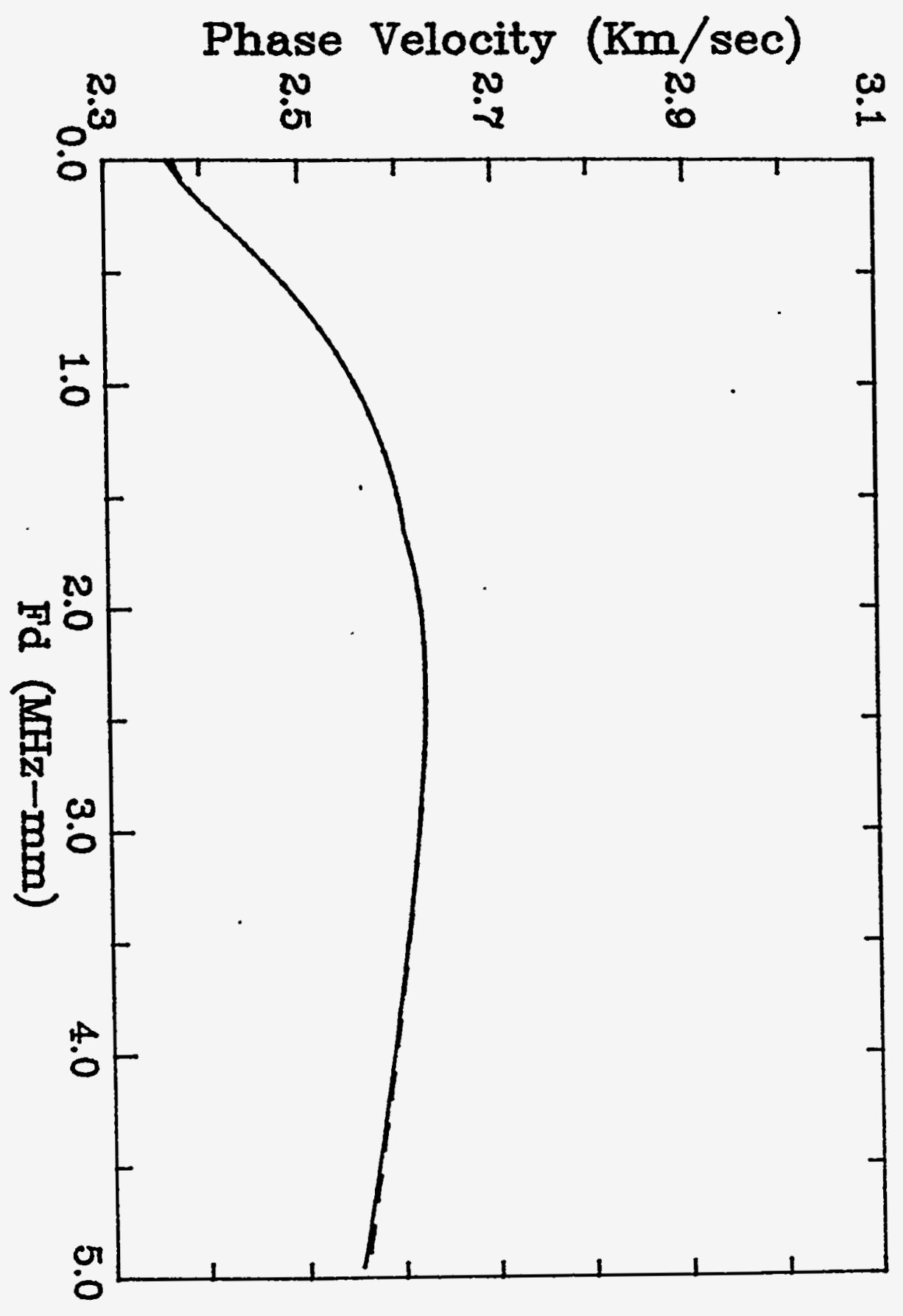

